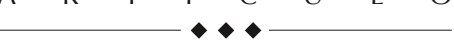

\title{
Modelización de materiales piezoeléctricos como generadores de energía
}

\author{
M. VÁZQUEZ RODRÍGUEZ', F. J. JIMÉNEZ MARTíNEZ', J. DE FRUTOS² \\ ${ }^{1}$ Dep. Sistemas Electrónicos y de Control, EUITT-UPM. Ctra. Valencia Km 7. 28031 Madrid, España. \\ 1,2 POEMMA-CEMDATIC. ETSIT-UPM. Ciudad Universitaria s/n. 28040 Madrid, España.
}

\begin{abstract}
En este trabajo se presenta el estudio realizado para determinar los modelos eléctricos equivalentes de materiales de tipo piezoeléctrico, para evaluar su capacidad como elementos generadores de energía eléctrica. Los diferentes modelos desarrollados a partir de los resultados experimentales registrados, se han utilizado para obtener la estructura óptima de elementos semiconductores a utilizar en la etapa de entrada del sistema de generación y acumulación de energía eléctrica, teniendo en cuenta su morfología, tipo de semiconductor a utilizar y número necesario, así como su comportamiento ante una demanda variable de potencia a suministrar
\end{abstract}

Palabras clave: Material piezoeléctrico, modelo eléctrico, generación de energía

\section{Power generator models of piezoelectric materials}

This paper presents the method to determine electrical equivalent models of piezoelectric materials used as electric power generating elements. The models developed from the experimental results have been used to obtain the type, amount and optimal topological structure of semiconductor elements needed in the input stage of the power generation system, and its behaviour under variable power supply demand.

Keywords: Piezoelectric material, electric model, power generation

\section{INTRODUCCIÓN.}

Proporcionar energía eficiente y limpia es un reto tanto para dispositivos microelectrónicos como convencionales. En los últimos años ha habido avances significativos en el desarrollo de fuentes de alimentación tales como cámaras de combustión, células solares, pilas de combustible, dispositivos termoeléctricos o microbaterías, la generación suministro y consumo de energía siguen siendo un obstáculo en el avance de nuevos dispositivos, como por ejemplo las nuevas MEMS como elementos complementarios a los CMOS puesto que el avance implicaría alejarse de la Ley de Moore que relaciona la capacidad de almacenamiento con el tamaño del dispositivo y su consumo de energía. Pero el reto de la energía no se limita a los dispositivos a microescala donde tanto el tamaño como la masa de las fuentes de alimentación convencionales, actúan como barreras de avance para el desarrollo de dispositivos electrónicos miniaturizados. De hecho, el sector industrial del transporte y los sectores comerciales siguen siendo áreas críticas de investigación en el consumo de energía para dispositivos convencionales, motivado en gran parte por el fuerte incremento del precio del petróleo, los efectos del calentamiento global y la contaminación.

A pesar de los notables avances en el desarrollo y la evaluación de tecnologías de energía sostenible en procesos macroscópicos (por ejemplo, células solares, hidroeléctrica, eólica, biocombustibles y geotermia), aproximadamente el $86 \%$ de la energía consumida por los Estados Unidos se deriva de los combustibles fósiles, y sólo el $14 \%$ procede de energías renovables (EE.UU. Departamento de Energía de EE.UU. y la Agencia de Protección del Medio Ambiente, 2005), porcentaje que en Europa es algo mayor sin llegar al 20\% y lejos de reducirse, el consumo de energía en el mundo, aumenta de manera continua.

En la figura 1, se representan diversos sistemas de captación de energía limpia que se están utilizando como alternativa a las energías fósiles (1-9). Para una mejor comparación de la fuente de generación de energía, se representa la densidad de potencia en función de la tensión generada. El tipo de fuentes consideradas corresponde a las pilas de Litio e IonLitio, celdas solares, celdas de combustible, dispositivos termoeléctricos, dispositivos termo-fotovoltaicos, dispositivos piezoeléctricos, y dispositivos electromagnéticos.

Los dispositivos piezoeléctricos presentan valores de densidad de potencia que son comparables a otras tecnologías de generación de energía, tales como las baterías de ion-litio y los generadores de potencia electromagnéticos, pero tienen menor densidad de potencia que las celdas solares avanzadas (alta eficiencia: aprox. $25 \%$ de 100-1000 AM intensidad solar simulada $\left(\mathrm{mW} / \mathrm{cm}^{2}\right)$. 
La línea de investigación en el aprovechamiento de esta fuente de energía, más que definir cuál de las diferentes formas alternativas de generar y almacenar energía es mejor, o si de manera más específica, sobre qué fuentes de energía tiene ventajas la generación de energía basada en el aprovechamiento de vibraciones y presiones, lo que se busca es determinar para qué aplicaciones esta tecnología puede aplicarse y sugerir áreas que necesitan avances en la investigación de dispositivos piezoeléctricos generadores de energía. En este sentido, en la tabla I, se proporciona información sobre diferentes tipos de aplicaciones de captación de energía piezoeléctrica, aplicación de la misma, y los resultados conseguidos referidos en la bibliografía.

De forma complementaria a lo recogido en la tabla anterior, en los últimos años se ha despertado un gran interés por el aprovechamiento dela energía mecánica y de impacto del tráfico rodado en carreteras y autopistas. Gran parte de este interés está motivado por la necesidad de disponer de dispositivos sensores autónomos y la necesidad de realizar sistemas de medida y control autoalimentados para monitorización de estructuras y grandes obras de ingeniería civil $(24,25)$. Uno de los problemas fundamentales a la hora de conseguir el aprovechamiento energético es disponer de dispositivos que aprovechen al máximo la configuración de los mismos y permitan su uso como parte activa de fuentes de alimentación. El primer obstáculo importante a salvar para realizar este estudio, es disponer de un modelo eléctrico equivalente de dichos materiales piezoeléctricos, que responda correctamente a la excitación provocada por el tráfico vial, fuente de energía mecánica generadora. Nos planteamos como objetivo en este trabajo la simulación del proceso de conversión de energía mecánica en eléctrica haciendo uso de materiales

\section{TABLA I. Cogeneración Piezoeléctrica}

\begin{tabular}{|c|c|c|c|c|c|c|c|}
\hline$\frac{\pi}{\stackrel{\pi}{d}}$ & 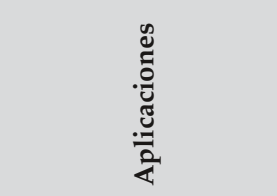 & 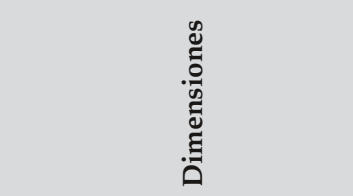 & 荧 & 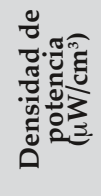 & $\frac{\sqrt[0]{3}}{\frac{\pi}{2}}$ & 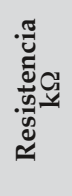 & 胥 \\
\hline $\begin{array}{l}\text { Composites de PZT, } \mathrm{d}_{33} 39 \mathrm{MPa} \\
\text { compresión }\end{array}$ & Composite & & & 12000 & & & 2,3 \\
\hline $\mathrm{PVF}_{2}$ Compresión & Rotor de aerogenerador & $500 \mu \mathrm{m}^{*} 90 \mathrm{~mm}^{*} 70 \mathrm{~mm}$ & 2,4 & 0,76 & 1 & 400 & 10 \\
\hline BIMORFO PVDF. Modo $_{31}$ & Elemento en zapatos & & 1300 & & 18 & 250 & 11 \\
\hline PZT DIMORFO, d31 & Elemento en zapatos & & 8400 & & 64,8 & 500 & 11 \\
\hline $\begin{array}{l}\text { PVDF bimorfo. Rotor de } \\
\text { aerogenerador. } 12 \text { cantilevers } \\
\text { bimorfos. Carga } \mathrm{d}_{31} \text { rotor }\end{array}$ & Rotor de aerogenerador & Cada bimorfo $60^{*} 20^{*} 0,5 \mathrm{~mm}$ & 10200 & 1420 & 6,8 & 4,6 & 12 \\
\hline Estructura rectangular de PZT & Implante de rodilla & $1^{*} 1 * 1,8 \mathrm{~cm}^{3}$ & 4800 & 890 & & & 14 \\
\hline $\begin{array}{l}\text { Membrana transductora } \\
\text { sometida a la vibración de un } \\
\text { agitador }\end{array}$ & $\begin{array}{l}\text { Sensores de baja } \\
\text { potencia }\end{array}$ & $\begin{array}{l}\text { Radio total: } 20,5 \mathrm{~mm}(12,5 \mathrm{~mm} \\
\text { de radio PZT), espesor } 230 \mu \mathrm{m} \text {, } \\
\text { Espesor de sujeción: } 400 \mu \mathrm{m}\end{array}$ & 1700 & 106 & 9 & 47 & 2,3 \\
\hline $\begin{array}{l}\text { Lámina delgada de PZT. } \\
\text { Generador Cantilever }\end{array}$ & MEMS & $\left(170^{*} 260\right) \mu \mathrm{m}^{2}$ & 1 & 740 & 2,2 & 5200 & 15 \\
\hline Lámina gruesa de PZT a $80 \mathrm{~Hz}$ & MEMS & Capas de $20 \mu \mathrm{m}$ & 2 & & 0,81 & 333 & 16 \\
\hline $\begin{array}{l}\text { PZT con 1,5 MPa tensión lateral } \\
\text { a } 15 \mathrm{~Hz}\end{array}$ & $\begin{array}{l}\text { Trasmisor autónomo } \\
\text { wireless }\end{array}$ & Volumen: $0,2 \mathrm{~cm}^{3}$ & 1200 & 6000 & 9 & & 17 \\
\hline $\begin{array}{l}\text { Generador de apilamiento de } \\
\text { PZT( } 164 \text { capas a } 1 \mathrm{~Hz} \text { y } 250 \mathrm{~N})\end{array}$ & $\begin{array}{l}\text { Potencia de activación } \\
\text { del músculo }\end{array}$ & $5^{*} 5^{*} 80 \mathrm{~mm}^{3}$ & 690 & 345 & 19,3 & 540 & 2,3 \\
\hline $\begin{array}{l}\text { Membrana de PZT en lámina } \\
\text { delgada acoplada al calor del } \\
\text { motor. }\end{array}$ & Híbrido & $\begin{array}{l}\text { Sup: } 3 \mathrm{~mm}^{2} \\
\text { Esp: } 3,4 \mu \mathrm{m}\end{array}$ & 56 & $5,510^{6}$ & & & 18 \\
\hline $\begin{array}{l}\text { Dispositivo címbalo con carga } \\
\text { d33 }\end{array}$ & Címbalo & $\begin{array}{l}\text { Diam: } 29 \mathrm{~mm} \\
\text { Esp: } 1 \mathrm{~mm}\end{array}$ & 39000 & 43900 & & 400 & 19 \\
\hline $\begin{array}{l}\text { Pulsador PZT (ignición) carga } \\
\text { d33 }\end{array}$ & Generador de pulsos & $\begin{array}{l}\text { Diam:1,27 cm } \\
\text { Esp 1,3 mm }\end{array}$ & $2510^{9}$ & $1,5110^{6}$ & 500 & 0,01 & 20 \\
\hline $\begin{array}{l}\text { PMN-PT composites. d } 33 \text { carga } \\
\text { comprensiva de } 40,4 \mathrm{MPa}\end{array}$ & Composite & & & 22100 & & & 21 \\
\hline $\begin{array}{l}\text { PZT entre acero y latón } \\
\text { sometidos a ciclos de tensión de } \\
0,7 \mathrm{~N} \text { a } 590 \mathrm{~Hz}\end{array}$ & & $0,51 \mathrm{~cm}^{3}$ & 11000 & 21570 & 14,07 & 18 & 22 \\
\hline $\begin{array}{l}\text { Arrays de nanohilos de } \\
\text { piezoeléctrico y } \mathrm{ZnO} \\
\text { semiconductor }\end{array}$ & $\begin{array}{l}\text { Biosensores } \\
\text { y electrónica } \\
\text { autoalimentada }\end{array}$ & $\begin{array}{l}300 \text { nm diámetro hilo. } \\
\text { Arrays de } 6,5^{\star} 3,2 \mu \mathrm{m}^{2}\end{array}$ & $\begin{array}{l}\sim 10-20 \mathrm{pW} \text { por } \\
\text { hilo }\end{array}$ & $100-200$ & 0,02 & & 23 \\
\hline
\end{tabular}


piezoeléctricos enterrados bajo la calzada puesto que es el escenario más probable de uso. A pesar de que mediante esta disposición se atenúa la transmisión de la presión de rodadura ejercida por los diferentes vehículos, se conjugan ventajas evidentes como son la menor fatiga de dichos materiales, la no alteración del confort de los usuarios de los vehículos y de ser así difícilmente manipulables o alterables de forma intencional o casual. En estas condiciones, se pretende simular el circuito de generación de energía asociado a diferentes distribuciones y configuraciones de los elementos a utilizar. En trabajos anteriores hemos presentado las características de un banco de ensayos que permite simular el tráfico rodado (26) y en consecuencia la forma y magnitud de la respuesta de los materiales piezoeléctricos sometidos a tráfico real. Así dispondremos de valores iniciales que nos permitirán alimentar y verificar el proceso de simulación de los diferentes tipos de configuración de los elementos piezoeléctricos, pudiendo determinar así la configuración más adecuada. La necesidad de asociar la respuesta del material a un circuito eléctrico más o menos complejo ha sido una constante en este tipo de aplicaciones. Desde considerar circuitos para el análisis de la potencia generada (27), considerar convertidores discontinuos en el modo de conducción $(28,31)$, convertidores electrónicos de potencia (29) o incluso circuitos adaptativos para aplicaciones remotas (30). En todos los casos se plantea el desarrollo de un circuito que ayude a explicar los fenómenos que tienen lugar. En nuestro caso, pretendemos, a partir de datos de caracterización determinados con el banco de simulación del tráfico rodado, plantear un modelo eléctrico del sistema que nos ayude a definir la configuración ideal de elementos, la forma en que han de conectarse, y los elementos de circuito destinados a conseguir la máxima eficiencia en el almacenamiento de la energía.

\section{MODELADO ELÉCTRICO DE CABLE PIEZOELÉCTRI- CO BAJO ESTÍMULO DE TRÁFICO RODADO.}

\subsection{Medida de las respuestas eléctricas con el banco de ensayos.}

Si planteamos la obtención de un modelo eléctrico equivalente para un compuesto piezoeléctrico que se ve sometido a la excitación mecánica provocada por la presión transmitida hacia el interior del pavimento por la tracción de las ruedas y la masa de los vehículos, nos enfrentaremos con un problema enormemente complejo especialmente si se asume tráfico real, con sus condiciones de contorno no lineales y la evidente componente probabilística de la tipología de las fuentes mecánicas que excitarán a estos materiales. De acuerdo con lo comentado en trabajos anteriores (26), una de las grandes virtudes del banco de ensayos que hemos diseñado para realizar estas caracterizaciones es su comportamiento periódico, regular y totalmente predecible al permitir replicar

TABLA II. RESUMEN DE ENSAYOS SOBRE CABLE PIEZOELÉCTRICO: TIEMPOS CARACTERÍSTICOS ( $\Delta \tau, \Delta$ T) Y VELOCIDADES SIMULADAS (V), SEGÚN VELOCIDAD ANGULAR DEL EJE DE SALIDA DE LA PLATAFORMA $\left(\mathrm{n}_{\mathrm{o}}\right.$ ) Y DEL ÁNGULO ENTRE EJES DE RODADURA ( $\beta$ ) PARA VEHÍCULO AUTOMÓVIL BERLINA DE 2,64 M DE BATALLA.

\begin{tabular}{|c|c|c|c|c|c|c|}
\hline $\begin{array}{c}\text { Ángulo entre ejes } \\
\beta\left(\left(^{\circ}\right)\right.\end{array}$ & $\begin{array}{l}N^{o} \text { de } \\
\text { ensayo }\end{array}$ & $\Delta \tau(\mathrm{s})$ & $\Delta \mathrm{T}(\mathrm{s})$ & $n_{\mathrm{o}}=(30 / \Delta T)(\mathrm{rpm})$ & $v=((2,64 \times 3,6) / \Delta \tau)(\mathrm{km} / \mathrm{h})$ & $\begin{array}{c}\text { Tensión de pico máximo } \\
\text { â (mV) }\end{array}$ \\
\hline \multirow{7}{*}{22} & 1 & 0,156 & 1,284 & 23,364 & 60,923 & 538,531 \\
\hline & 2 & 0,136 & 1,148 & 26,132 & 69,882 & 531,581 \\
\hline & 3 & 0,120 & 1,000 & 30,000 & 79,200 & 643,253 \\
\hline & 4 & 0,108 & 0,892 & 33,632 & 88,000 & 647,536 \\
\hline & 5 & 0,096 & 0,804 & 37,313 & 99,000 & 779,142 \\
\hline & 6 & 0,088 & 0,736 & 40,761 & 108,000 & 821,405 \\
\hline & 7 & 0,082 & 0,674 & 44,510 & 115,902 & 794,013 \\
\hline \multirow{6}{*}{45} & 8 & 0,332 & 1,34 & 23,388 & 28,627 & 476,660 \\
\hline & 9 & 0,284 & 1,148 & 26,132 & 33,465 & 454,793 \\
\hline & 10 & 0,248 & 1,000 & 30,000 & 38,322 & 549,714 \\
\hline & 12 & 0,196 & 0,800 & 37,500 & 48,490 & 723,335 \\
\hline & 13 & 0,178 & 0,734 & 40,872 & 53,393 & 818,550 \\
\hline & 14 & 0,168 & 0,674 & 44,510 & 56,571 & 900,096 \\
\hline \multirow{7}{*}{90} & 15 & 0,680 & 1,36 & 22,059 & 13,976 & 551,763 \\
\hline & 16 & 0,576 & 1,156 & 25,952 & 16,500 & 675,318 \\
\hline & 17 & 0,500 & 1,004 & 29,880 & 19,008 & 743,948 \\
\hline & 18 & 0,444 & 0,888 & 33,784 & 21,405 & 770,583 \\
\hline & 19 & 0,404 & 0,806 & 37,221 & 23,525 & 818,952 \\
\hline & 20 & 0,368 & 0,732 & 40,984 & 25,826 & 886,554 \\
\hline & 21 & 0,340 & 0,676 & 44,379 & 27,953 & 847,158 \\
\hline
\end{tabular}


experimentos con resultados contrastables ante consignas idénticas. Así pues, asumiendo tráfico continuo, somos capaces de abrir un camino, que se detalla en este punto, y arbitrar un procedimiento para obtener modelos lineales válidos para el estudio que sobre aprovechamiento energético interesa realizar, abierto a cualquier otro compuesto que posea propiedades piezoeléctricas, al margen del cable usado en estas primeras experimentaciones.

Se han realizado ensayos sobre cables piezoeléctricos, disponiéndolos siguiendo los correspondientes diámetros de la plataforma estática. En la figura 2 se presenta la forma de onda tipo registrada en los ensayos efectuados. En ella se ha resaltado el intervalo temporal $\Delta \mathrm{T}$, que representa el período de repetición eléctrico de las señales recogidas que simulan el paso repetitivo de un vehículo de dos ejes, $\Delta \tau$ que es el intervalo temporal entre los estímulos recogidos debidos a cada eje de un único vehículo y finalmente â que representa su valor de tensión de pico máximo. El registro de datos, se ha llevado a cabo con un el osciloscopio TDS7054 de Agilent Technologies, con sonda de prueba 10073C (10:1, $500 \mathrm{MHz}$ BW) y seleccionando $1 \mathrm{M} \Omega$ como impedancia de entrada, tanto para

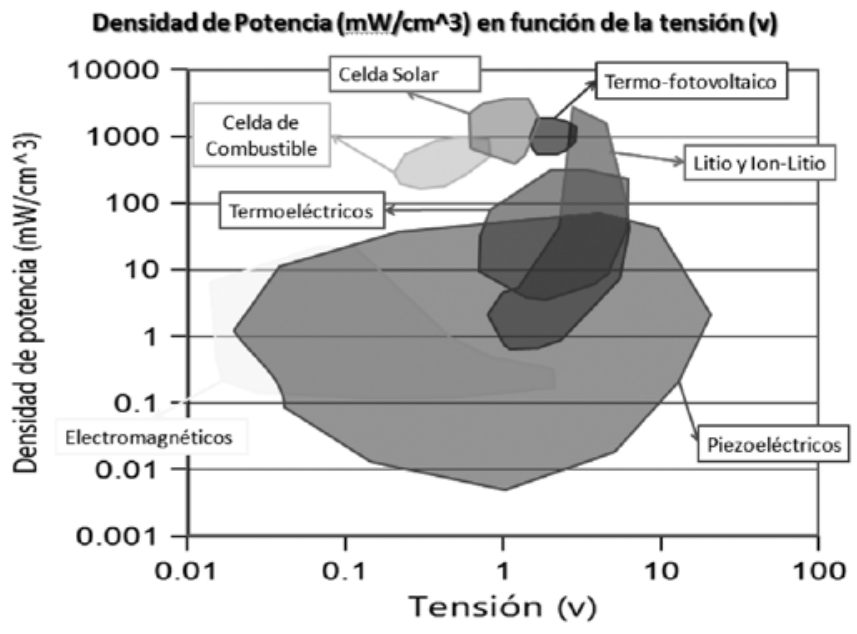

Figura 1: Representación de la densidad de potencia en función del voltaje de nuevas tecnologías de generación de energía limpia. (2)

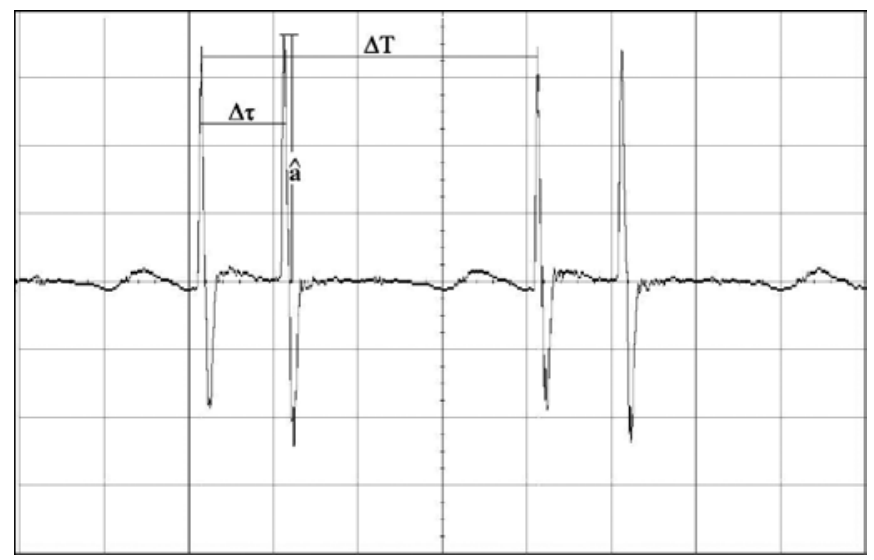

Figura 2: Señal eléctrica tipo registrada de un cable piezoeléctrico mediante el banco de ensayos. los ensayos correspondientes a velocidades mínimas como para las máximas. En la tabla II se presenta el resumen de los ensayos efectuados con las tres configuraciones mecanizadas en el banco de ensayos para $22^{\circ}, 45^{\circ}$ y $90^{\circ}$ de ángulo relativo (definido como $\beta$ ) entre los dos ejes disponibles actualmente. Esto ha permitido disponer de ensayos que comprenden desde una velocidad equivalente próxima a $15 \mathrm{~km} / \mathrm{h}$, hasta un entorno próximo a $120 \mathrm{~km} / \mathrm{h}$.

Por otra parte, es importante dejar claro que será el ángulo relativo entre ejes de la plataforma giratoria $\beta\left(^{\circ}\right)$ junto con la velocidad angular de la misma $n_{o}(r p m)$, lo que permite simular el tiempo entre impactos de los ejes $\Delta \tau$ (s) de un vehículo real tal y como resume la ecuación [1] y particulariza la tabla II.

$$
\Delta \tau=\frac{\beta}{n_{o} \cdot 6}
$$

La ecuación [2] permite obtener la velocidad equivalente $\mathrm{v}$, expresada en $\mathrm{km} / \mathrm{h}$, de un vehículo simulado cuya separación entre sus ejes delantero y trasero (batalla) se expresa con el parámetro b $(\mathrm{cm})$. En la tabla II se ha presentado la velocidad simulada para un vehículo de $b=264 \mathrm{~cm}$ en los diferentes ensayos realizados.

$$
v=\frac{b}{\Delta \tau \cdot 100} \cdot 3,6
$$

Como consecuencia de este primer análisis sobre los datos registrados y buscando obtener el mayor aprovechamiento energético de la carga liberada en el cable piezoeléctrico, desarrollaremos un modelo eléctrico que replique el comportamiento del cable como generador eléctrico, excitado mecánicamente con el banco de ensayos, en las condiciones en que se consigue mayor amplitud instantánea en la señal eléctrica recogida.

\subsection{Metodología para la obtención de los modelos eléctricos equivalentes.}

Una vez que se han obtenido las respuestas eléctricas, planteamos la síntesis del modelo eléctrico equivalente del material piezoeléctrico. El modelo que refleja el comportamiento eléctrico de un material piezoeléctrico sometido a excitación mecánica es el formado por la asociación serie de un generador de tensión con la capacidad equivalente del material o, alternativamente, su equivalente Norton, mediante fuente de corriente en paralelo con dicha capacidad equivalente.

La forma de onda de la respuesta eléctrica del cable piezoeléctrico, sometido a estímulo equivalente a tráfico rodado, se ha obtenido al aplicar entre sus dos terminales una sonda de medida conectada a un canal de un osciloscopio, este método representa una carga conectada al cable de 1 $\mathrm{M} \Omega$, según se ha detallado anteriormente. Partiendo de la medida registrada debemos encontrar el modelo interno del piezoeléctrico que actuando como generador incorpora también una impedancia serie de tipo capacitivo. Nuestro trabajo consiste en obtener el modelo eléctrico del cable que aplicado a una carga del mismo valor produzca exactamente la misma forma de onda registrada en el osciloscopio y nos permita experimentar mediante simulaciones cuál será 
su comportamiento cuando el piezoeléctrico se conecte a circuitos de acumulación de carga eléctrica.

En este método asumimos que la excitación mecánica periódica proporcionada por el banco de ensayos se corresponderá con un tráfico continuo de vehículos, asî cumplimos la condición de periodicidad en la señal que permite aplicar el desarrollo en serie de Fourier a la señal eléctrica medida y obtener su modelo lineal equivalente, a partir del cual y teniendo muy en cuenta la capacidad serie y la impedancia de carga con la que se ha registrado la medida, podremos calcular el equivalente como generador eléctrico buscado. Por tanto, para cada uno de los ensayos efectuados se ha realizado el siguiente procedimiento.

- Extracción de los valores de amplitud y tiempo de cada ensayo en formato de fichero de texto con los valores correspondientes a un período eléctrico $\Delta \mathrm{T}$.

- Obtención de las componentes de Fourier de la señal registrada de cada ensayo, hasta el armónico necesario, usando un generador de tensión VPWL_F_RE_FOREVER con el fichero de datos tipo texto anterior, mediante la herramienta incorporada en el programa de simulación electrónica PSpice, sobre la frecuencia central dada por $\mathrm{f}_{\mathrm{o}}$ $=(1 / \Delta \mathrm{T})$.

- Comprobación de que el número de componentes de Fourier elegidas reproduce la señal eléctrica original de partida. Para ello se construye la asociación serie de los generadores sinusoidales de tensión obtenidos mediante el desarrollo de Fourier y se representa junto con el generador VPWL_F_RE_FOREVER, cargando ambos en condiciones próximas a circuito abierto (1000 G $\Omega$ en el simulador).

- Obtención del modelo real mediante la asociación serie de los generadores internos equivalentes y el condensador equivalente serie del material piezoeléctrico. Para ello se aplica el principio de superposición realizando el cálculo a cada frecuencia de dichos generadores sinusoidales internos y utilizando el valor de la impedancia que presenta la capacidad del piezoeléctrico a dichas frecuencias.
- Validación del modelo eléctrico real con carga de 1 $\mathrm{M} \Omega$, equivalente a la sonda del osciloscopio utilizada en la adquisición de las respuestas eléctricas reales, por comparación con la gráfica disponible de los datos de partida procedentes de los ensayos (generador VPWL_F_ RE_FOREVER inicialmente utilizado).

La realización de este proceso, aplicado a cada uno de los ensayos, nos conduce a la utilización de 75 generadores sinusoidales en serie con la capacidad del material, consecuencia del desarrollo en serie de Fourier calculado para cada ensayo, debiendo respetar el número completo de posiciones decimales que nos entrega la herramienta de cálculo del simulador para evitar la generación de un ruido eléctrico acumulativo con el tiempo que se produce si se truncan decimales. Con ayuda de las figuras 3 y 4 se ilustra este método. En la figura 3 se representa la señal recogida en el ensayo número 14 de la tabla II y el modelo lineal equivalente obtenido con el procedimiento descrito anteriormente, se presenta el detalle de los valores de amplitud, frecuencia y ángulo de fase correspondientes al desarrollo en serie de Fourier. Se puede observar que el generador V1 posee una frecuencia $f_{1}=(1 / \Delta T)=1,483679 \mathrm{~Hz}$, valor correspondiente con toda la precisión disponible de los datos de tensión y tiempo registrados. En la figura 4 se presenta el resultado final del procedimiento al comparar la señal introducida al simulador correspondiente a este ensayo y la producida por el modelo equivalente final representado mediante el generador V57_45 (cuya descomposición jerárquica se corresponde con la figura $3(\mathrm{~b})$ ), sobre una carga de $1 \mathrm{M} \Omega$, que reproduce el efecto de la sonda de prueba del osciloscopio utilizado para su adquisición. La comparación de ambas señales presenta una altísima coincidencia tanto en forma de onda como en valores instantáneos, habiéndose comprobado que con el desarrollo hasta el armónico 75 podemos reproducir (en las mismas condiciones de carga de los ensayos) la forma de onda de la señal original, tolerando un pequeño error en las oscilaciones mecánicas que provoca el funcionamiento del banco de ensayos y que no contribuyen al estudio sobre acumulación de carga que describimos a continuación.

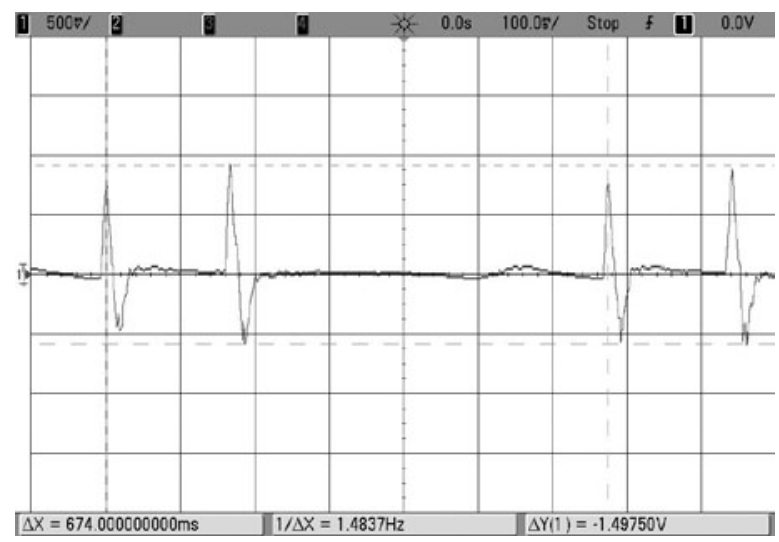

a)

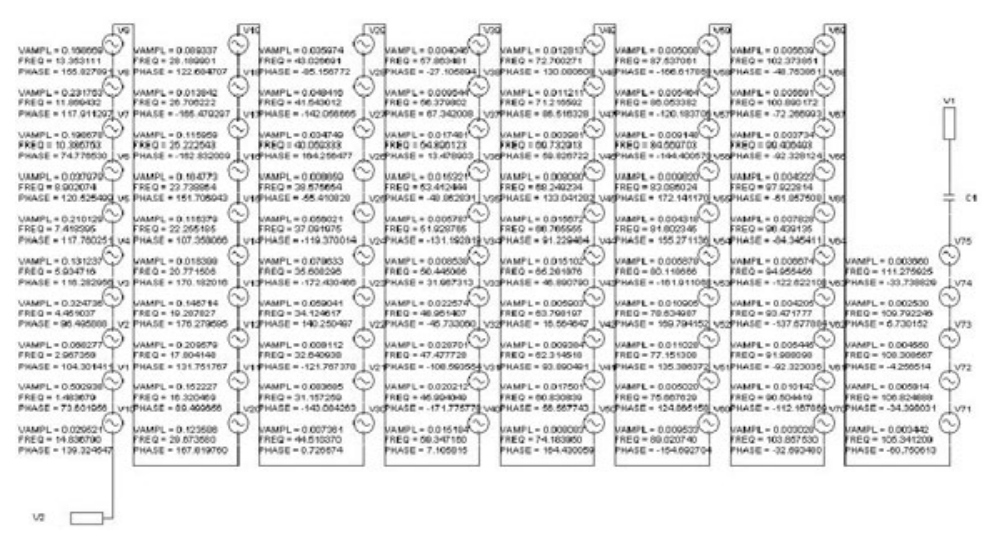

b)

Figura 3: (a) Tensión recogida del ensayo 14 de la tabla II. (b) Modelo eléctrico equivalente para este ensayo. 


\section{USO DEL MODELO PARA EVALUAR LA OPTIMIZA- CIÓN DEL APROVECHAMIENTO ENERGÉTICO.}

El estudio se centra a continuación en la utilización de los modelos lineales equivalentes obtenidos para cuantificar y optimizar el método de acumulación de la carga liberada. Abordamos este estudio planteando que la disposición de los cables de tipo piezoeléctrico que actuarán como generadores de carga se hallarían de forma consecutiva bajo el pavimento. En los ensayos efectuados, tomando un número reducido de estos cables, se ha comprobado como la respuesta eléctrica individual era equiparable si se distancian al menos $1,6 \mathrm{~cm}$ entre sí. La inclusión en el modelo equivalente de este efecto se realiza introduciendo a cada uno de los generadores que modelan un cable un desplazamiento temporal según la ecuación 3.

$$
t_{D}=\frac{d_{c}}{n_{o} \cdot(\Pi / 30) \cdot R}
$$

Esta ecuación determina el tiempo de retardo en segundos $\left(t_{D}\right)$ entre dos excitaciones producidas por sendos cables consecutivos a una distancia entre ellos $d_{c}$ (expresada en metros), cuando dichas excitaciones se producen en condiciones de tráfico continuo para un régimen de velocidad equivalente correspondiente con la velocidad de revolución $\mathrm{n}_{\mathrm{o}}$ (rpm) del banco de ensayos y con radio de la plataforma giratoria $R(m)$, dado que $R=0,75 m>d_{c}=0,016 m$, usamos la ecuación 3 admitiendo la aproximación geométrica entre el arco y su cuerda.

La forma de acumular carga eléctrica se realiza mediante la utilización de un elemento capacitivo. La forma de conseguir
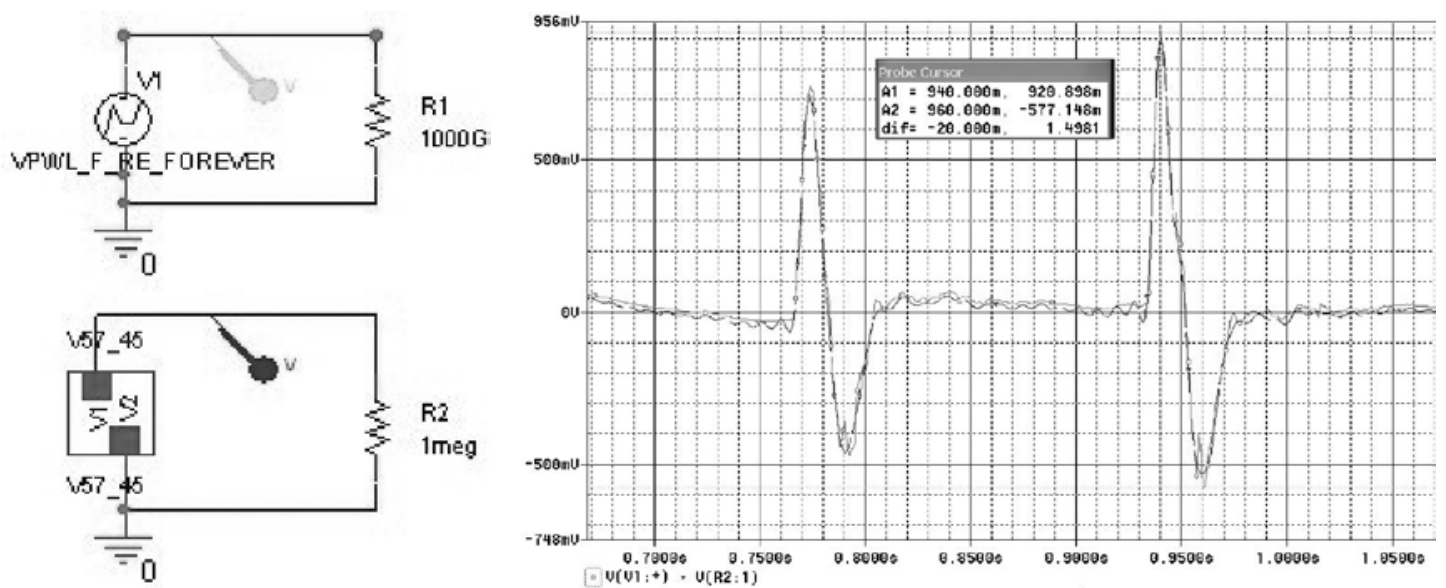

a)

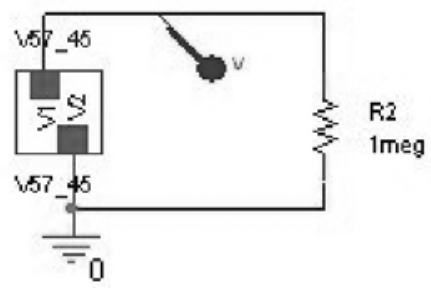

b)

Figura 4: Validación mediante simulación del modelo del ensayo 14 de la tabla II: (a) Circuitos simulados (b) Resultados de simulación.

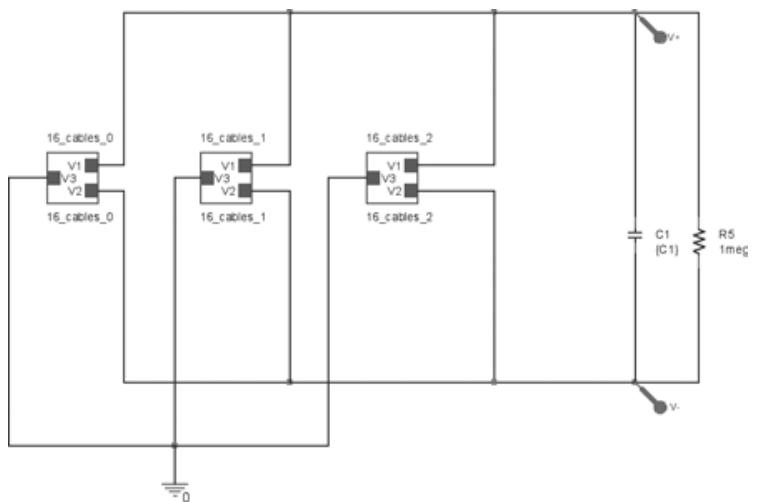

a)

Figura 5: (a) Estructura jerárquica de topología rectificadora polifásica en estrella de 48 puntas. (b) Detalle de un bloque de 16 cables generadores en topología polifásica de estrella de 16 puntas. 
trasvasar la carga generada por el efecto piezoeléctrico al elemento almacenador de carga se basará en la utilización de diodos semiconductores, de forma que se consiga aprovechar tanto la carga liberada por el efecto de la presión transmitida al iniciar el contacto con el punto bajo el que se encuentra el cable, como la liberación de carga en oposición al producirse la relajación de esta presión. La topología de la estructura de semiconductores y el tipo más adecuado entre ellos se trata a continuación.

\subsection{Estructuras rectificadoras para el almacenamiento de la carga. Topologías y comparativa de semiconductores.}

Se inicia a continuación el estudio comparativo entre diferentes topologías rectificadoras y a su vez según el tipo de diodo semiconductor a emplear en ellas, con el objetivo de almacenar la mayor carga posible de la generada en el compuesto piezoeléctrico. Para poder realizar esta comparativa se van a realizar las correspondientes simulaciones electrónicas utilizando el simulador PSpice disponible en la Suite de diseño electrónico $\mathrm{OrCAD}^{\circledR}$ de la firma Cadence Design Systems, Inc., herramienta ampliamente utilizada y de reconocido prestigio dada la calidad de los modelos funcionales de los diferentes semiconductores que implementa y de su motor de cálculo de resolución de las ecuaciones circuitales.

El proceso seguido para recopilar la información se basa en emplear valores constantes tanto de la capacidad como de la resistencia terminal de carga. La modificación del valor de la capacidad sólo contribuye al tiempo que necesitará el circuito acumulador en alcanzar su valor de régimen permanente. Fijando el valor de la capacidad podemos utilizar un tiempo de simulación fijo, facilitando la agrupación de los resultados gráficos.

La contribución de varios cables actuando como generadores se ha resuelto mediante la utilización de estructuras rectificadoras basadas en puente de diodos, conectando un puente por cada cable generador y conectando a su vez estas estructuras básicas en paralelo, o bien mediante la variante topológica inspirada en sistemas polifásicos, mediante configuración en estrella multipunta. Se ha estudiado adicionalmente la topología multifásica poligonal, pero fue descartada muy pronto al conducir a resultados netamente peores que la homóloga en estrella, por lo que se presentarán estos resultados únicamente junto con los de las estructuras en puente. A modo de ejemplo, en la figura 5 (a) se presenta una estructura jerarquizada en estrella formada por tres bloques, detallándose su respectiva estructura para 16 cables de cada bloque en la figura 5(b), lo que representa un total de 48 cables generadores en asociación polifásica.

En la figura 6 se representan las estructuras jerárquica y de detalle para 48 cables generadores asociados en paralelo mediante la topología de puente de diodos.

En la figura 7 presentamos la evolución de la tensión en el condensador de acumulación de carga, agrupando los resultados en función de la tecnología de los semiconductores empleados y del tipo de topología rectificadora en la asociación de 48 cables generadores. Se han comparado los resultados para diodos rectificadores de silicio de propósito general (tipo 1N400X), de señal (tipo 1N4148) y de tipo Schottky (BAS40-04W y RB751). El tiempo de cada simulación en los ordenadores utilizados, dotados de microprocesador Intel ${ }^{\circledR}$ Core $^{\mathrm{TM}} 2$ E8400 a $3 \mathrm{GHz}$ y 2 GB de memoria RAM, ha sido

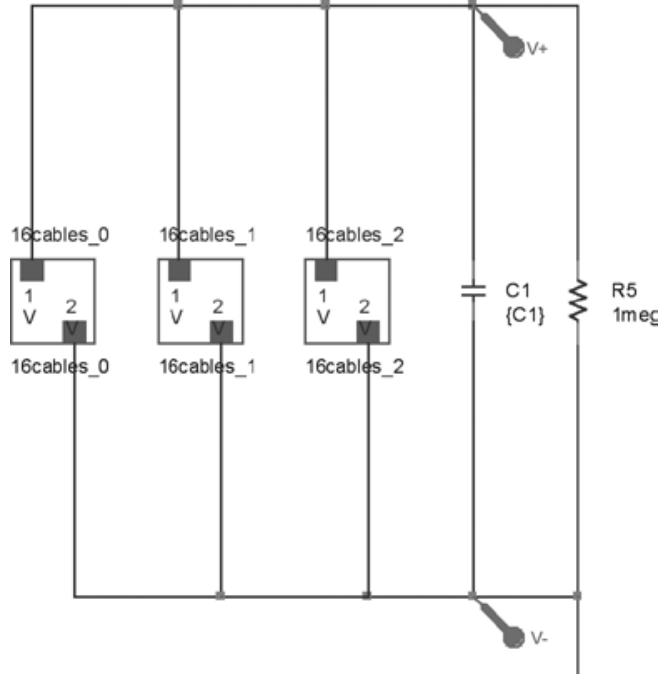

a)

b)

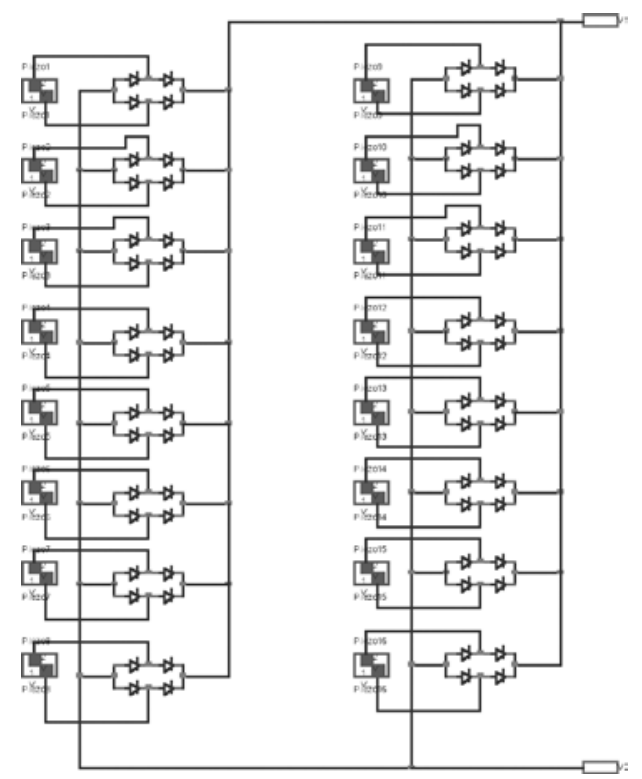

Figura 6: (a) Estructura jerárquica de rectificadores en puente para 48 cables generadores en paralelo (b) Detalle de un bloque de 16 cables generadores.

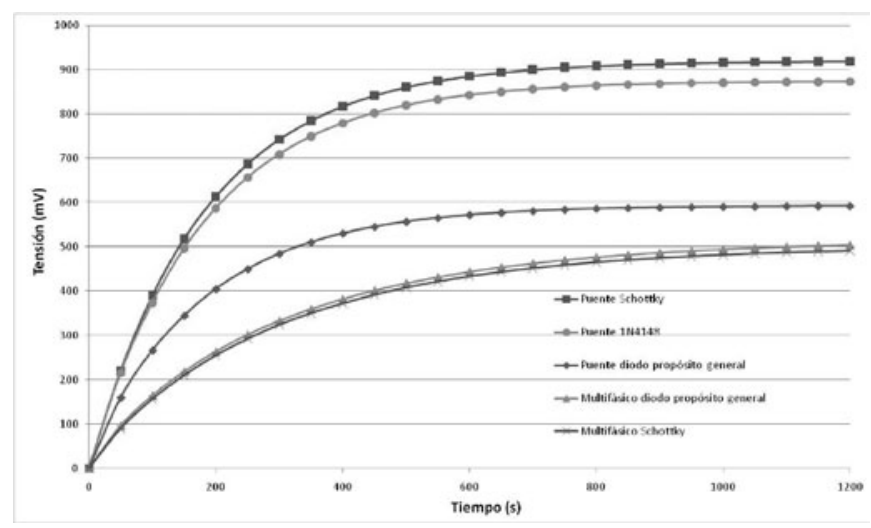

Figura 7: Tensión en el condensador de acumulación en función del tiempo de simulación, según topología rectificadora y tipo de diodo utilizado. 
TABLA III. TENSIÓN MÁXIMA CONSEGUIDA EN ASOCIACIONES EN SERIE DE CIRCUITOS ELEMENTALES DE N CABLES GENERADORES E INFLUENCIA DE LOS DIODOS DE RECIRCULACIÓN DE CORRIENTE

\begin{tabular}{c|c|c|c|c|c|c|c|c|c} 
Tensión final $(\mathbf{m V})$ & \multicolumn{1}{c}{ N=2 cables } & \multicolumn{1}{c}{$\%$} & \multicolumn{1}{c}{ N=4 cables } & $\%$ & \multicolumn{2}{c}{ N=5 cables } & $\%$ & \multicolumn{2}{c}{ N=8 cables } \\
\hline Sin diodo & 138,718 & 80,87 & 253,307 & 78,93 & 311,792 & 77,72 & 492,731 & 80,18 \\
\hline 1 diodo & 171,537 & 100 & 289,506 & 90,21 & 350,178 & 87,29 & 535,295 & 87,11 \\
\hline (N-1) diodos & 171,537 & 100 & 320,942 & 100 & 401,187 & 100 & 614,506 & 100 \\
\hline
\end{tabular}

de 26 horas en el caso de cada simulación que emplea diodos de tipo Schottky y de 16 horas para las correspondientes con diodos de señal. A la vista de la similitud entre el resultado final para ambos en la topología en puente y debido a la gran diferencia de tiempos de simulación se decidió continuar a partir de este momento para el resto de los estudios con el tipo de diodo $1 \mathrm{~N} 4148$

Finalmente, en la figura 8 presentamos los resultados globales que ilustran la tensión disponible según el número de circuitos contribuyendo en paralelo, para estructura rectificadora de puente de diodos tipo 1N4148. Con 80 circuitos en paralelo, se alcanza el máximo valor posible de tensión disponible en el condensador acumulador, obteniéndose una tensión disponible de 1.033,80 mV.

\subsection{Asociación serie de estructuras rectificadoras.}

Dado que la optimización de carga acumulada ha experimentado un máximo según los estudios anteriores y para poder alcanzar tensiones prácticas de alimentación para dispositivos o sistemas sensores autónomos se va a estudiar seguidamente la expansión, mediante la incorporación de nuevos cables generadores, de las estructuras paralelo anteriormente estudiadas conectándolas a su vez en asociación serie, determinando además la conveniencia de incluir algún camino de retorno de corriente de carga entre dichas estructuras serie que optimice todavía más este proceso.
Respecto a la conveniencia de disponer un condensador de acumulación por cada grupo paralelo de generadores o usar un único condensador para la asociación serie final, el conjunto de simulaciones realizadas indica esta última opción como óptima, se omiten los resultados obtenidos en beneficio de no alargar innecesariamente la cantidad de conclusiones presentadas.

Partimos de los modelos realizados para 80 cables generadores y pretendemos obtener el comportamiento de las diferentes posibilidades resultado de combinar, para un número creciente de asociaciones de circuitos en serie, los circuitos asociados en paralelo (que denominaremos circuitos elementales) que hemos visto en 3.1. Posteriormente, se estudió la idea de incluir en disposición antiparalelo con los circuitos elementales, algunos diodos que permitan una recirculación de las corrientes de carga y su efecto en número sobre la tensión conseguida en la capacidad que actúa como acumulador.

Gráficamente, en la figura 9 se presenta el ejemplo del caso formado por la asociación serie de circuitos elementales compuestos por $\mathrm{N}=8$ cables generadores con topología rectificadora en puente de diodos conectados en paralelo, y su asociación mediante 10 circuitos en serie. Se detalla en la figura 9 (c) la incorporación de N-1 diodos conectados en antiparalelo con los circuitos elementales para proporcionar un camino de recirculación de la corriente de retorno entre las estructuras en serie.

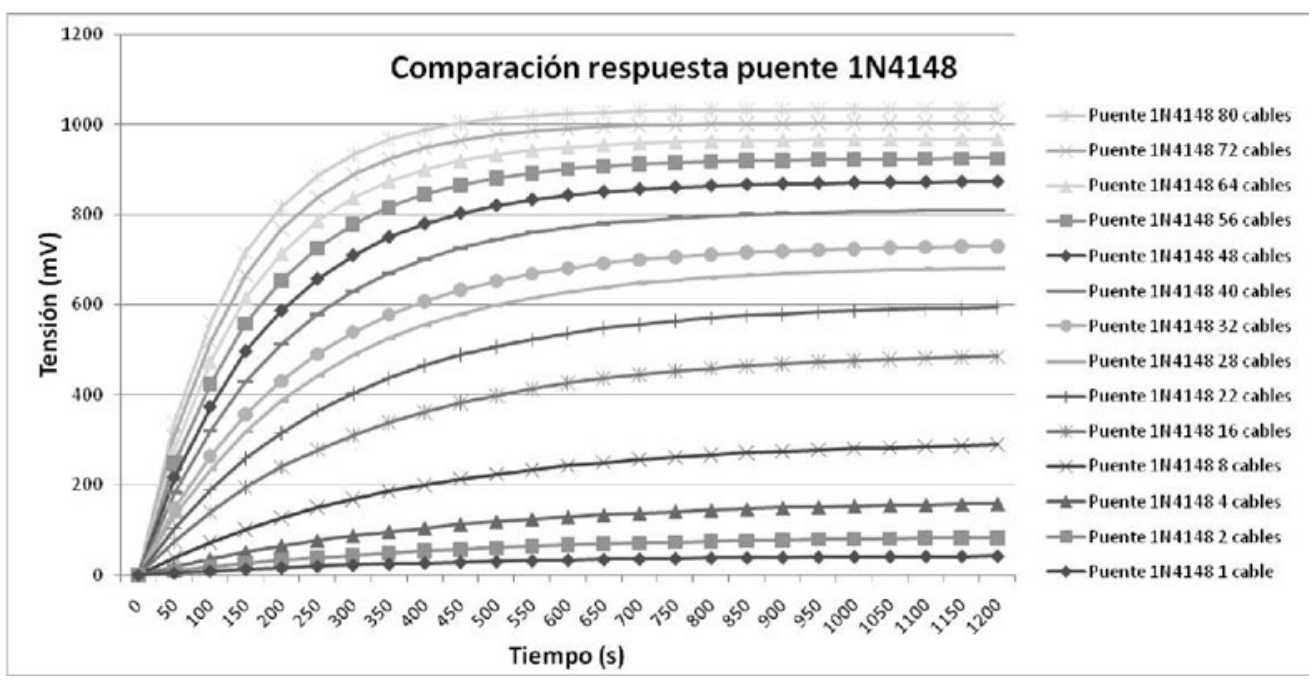

Figura 8: Gráficas comparativas de la tensión en el condensador en función del tiempo, según el número de circuitos rectificadores paralelo, con topología en puente, para diodos tipo $1 \mathrm{~N} 4148$. 
En la tabla III, se resumen los valores finales de tensión máxima conseguida combinando hasta 80 cables piezoeléctricos, mediante circuitos elementales de $\mathrm{N}=2,4,5$ y 8 cables generadores conectados en paralelo y el porcentaje que sobre el valor máximo posible (alcanzado en todos los casos para circuitos que utilizan $\mathrm{N}-1$ diodos de recirculación) se obtendría para el caso de no disponer ningún diodo en antiparalelo o para el caso de incluir tan sólo uno de ellos por cada circuito elemental paralelo. Se puede observar que si no se incluyen diodos de recirculación se pierde del orden de un $20 \%$ sobre la tensión alcanzable en todos los casos presentados.

\subsection{Curvas de regulación.}

A continuación se presenta el comportamiento de la tensión disponible en función del consumo de corriente que se solicite, así como el valor de potencia eléctrica disponible para su aplicación en un sistema autoalimentado. Para ello, se evalúan las sucesivas simulaciones modificando la resistencia terminal de carga, para la topología formada por dos circuitos elementales en serie. Cada circuito elemental se forma mediante la asociación en paralelo de 80 cables piezoeléctricos generadores con topología rectificadora en puente. Se han obtenido resultados para condición de circuito abierto y el detalle en torno al punto de máxima potencia con valores de resistencia terminal desde $1 \mathrm{M} \Omega$ hasta $100 \mathrm{k} \Omega$ (con decremento de valor constante de $100 \mathrm{k} \Omega$ ) y para valores de 90 $\mathrm{k} \Omega, 60 \mathrm{k} \Omega, 30 \mathrm{k} \Omega, 20 \mathrm{k} \Omega, 10 \mathrm{k} \Omega, 1 \mathrm{k} \Omega$ y $100 \Omega$. Cada una de las simulaciones genera un fichero de datos de $160 \mathrm{~Gb}$, por lo que se ha tenido que realizar de forma separada, ya que el uso del análisis paramétrico hubiera sido imposible efectuarlo por la limitación de la capacidad del disco duro de los ordenadores empleados.

En la figura 10 se presenta la curva de regulación obtenida, en la que se refleja la variación de la tensión continua disponible en el condensador acumulador para diferentes consumos de corriente dados por las resistencias de carga utilizadas. En la misma figura 10 se representa la potencia que es capaz de entregar esta estructura acumuladora. El punto de

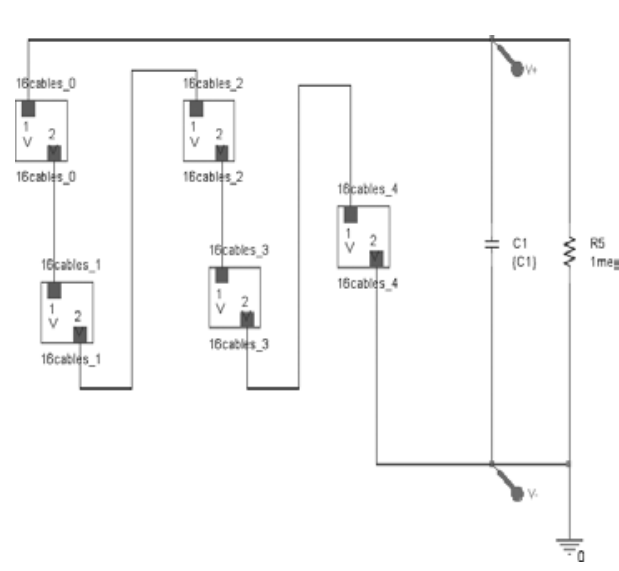

a)

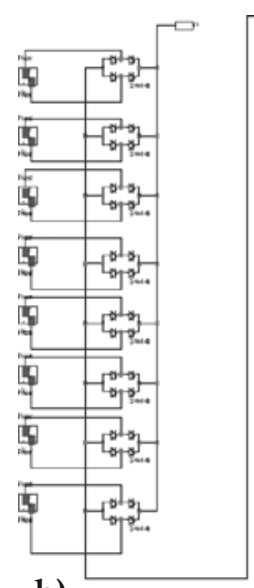

b)

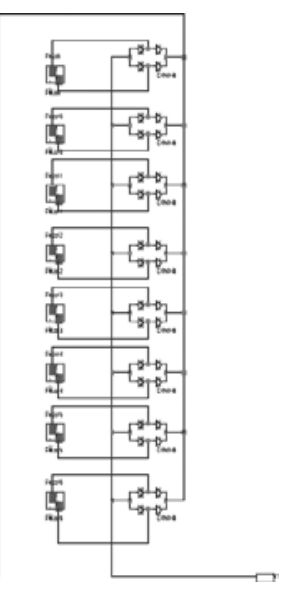

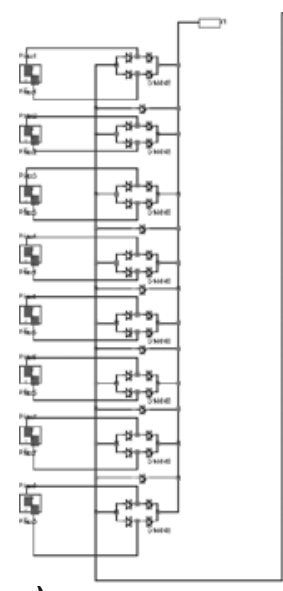

c)

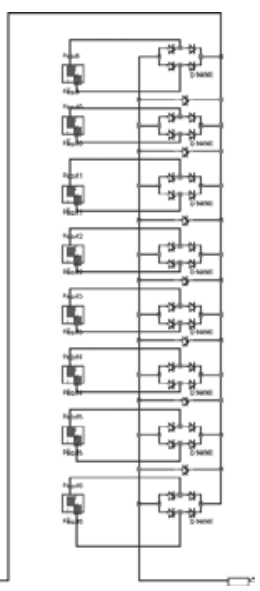

Figura 9: Asociaciones serie (a) 10 circuitos serie de 8 generadores paralelo cada uno, (b) Detalle de bloque jerárquico de (a) mediante asociación serie de 2 circuitos de 8 generadores paralelo cada uno, sin diodos de recirculación (c) Detalle de bloque jerárquico de (a) con incorporación de $\mathrm{N}-1$ diodos de recirculación
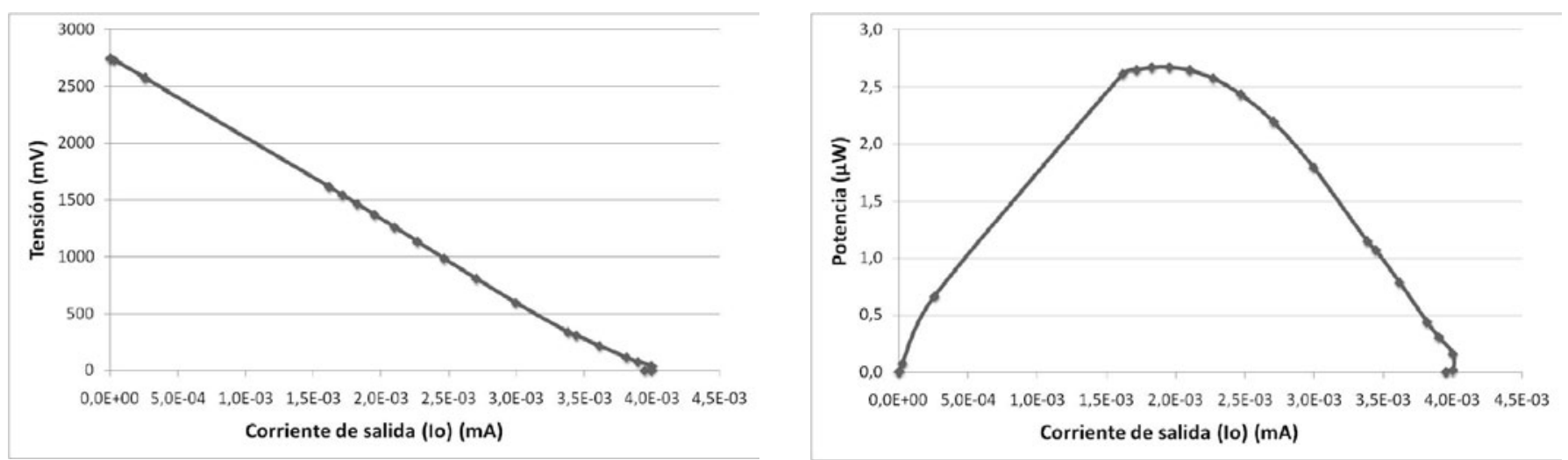

Figura 10: Resultados de la asociación de 2 circuitos serie formados por N=80 generadores en paralelo en cada uno: (a) Tensión disponible según la corriente consumida, (b) Potencia disponible según corriente consumida. 
funcionamiento que presenta un resultado optimizado para la estructura analizada es el obtenido para un consumo de $1,954 \mu \mathrm{A}$ en régimen continuo, para $1.367,9 \mathrm{mV}$ de tensión, liberando en estas condiciones una potencia de 2,67 $\mu \mathrm{W}$, correspondiente a la simulación con $\mathrm{R}=700 \mathrm{k} \Omega$.

\subsection{Respuesta con modelos correspondientes a ensayos amplificados mecánicamente.}

La configuración del banco de ensayos permite suplementar mediante peso adicional (26) las condiciones de excitación mecánica con las que se realizan los ensayos. Mediante la utilización de esta posibilidad se han realizado ensayos para obtener la curva de regulación y el punto de potencia optimizada si el tráfico continuo que estimulara a los cables piezoeléctricos enterrados fuera el de vehículos pesados. Para las mismas condiciones de velocidad aplicadas en el estudio realizado anteriormente para vehículos ligeros, que producen la máxima respuesta instantánea de tensión en el cable piezoeléctrico, se modela el cable generador para los ensayos con amplificación mecánica según el procedimiento descrito en 2.2. Se representan en la figura 11 las gráficas correspondientes a las simulaciones obtenidas, para las asociaciones paralelo desde 1 hasta 80 cables conectados mediante puentes rectificadores que utilizan diodos del tipo 1N4148, detallándose la diferencia entre los resultados presentados en 3.1 y los del nuevo modelo con amplificación mecánica, observando que la proporcionalidad entre ambos presenta, en valor promedio, un factor de 4 entre las simulaciones realizadas. Finalmente en la figura 12 se representa la gráfica de regulación y la potencia entregada en condiciones de consumo de corriente variable, para las simulaciones correspondientes a dos circuitos en serie de $\mathrm{N}=80$ cables generadores asociados en paralelo en cada uno, variando la resistencia de carga de igual modo a lo expuesto en 3.3. El punto de utilización que proporciona el valor óptimo para la potencia extraída con este método de aprovechamiento energético es el correspondiente con
$47,6 \mu \mathrm{W}$, en el que se entregaría una corriente de 8,246 $\mu \mathrm{A}$, proporcionando una tensión continua de $5.772,3 \mathrm{mV}$. Comparando con 3.3, observamos que la relación, para el punto óptimo conseguido para la simulación con $\mathrm{R}=700 \mathrm{k} \Omega$, entre las corrientes entregadas resulta ser de 4,22006 y para las tensiones de 4,21982, factores prácticamente coincidentes con la relación presentada entre ambos modelos para las simulaciones presentadas en la figura 11. La relación entre los valores de potencia para ambos estudios para el punto óptimo resulta ser de 17,82 , en lógica consonancia con al valor cuadrático que relaciona potencia con corriente (o con tensión) sobre la misma $\mathrm{R}$ de carga.

Comprobamos además que estos resultados verifican a su vez el principio de máxima transferencia de potencia, ya que la máxima potencia que se puede extraer de la fuente de energía piezoeléctrica modelada la expresa la ecuación 4. Esta potencia se obtendrá cuando la resistencia de carga $\left(R_{\text {out }}\right)$ sea igual a su resistencia de salida equivalente $\left(R_{o}\right)$, dónde $\mathrm{V}_{\mathrm{oc}}$ es el valor de la tensión en el condensador de acumulación de carga en condiciones de circuito abierto (idealmente resistencia de carga infinita) y $R_{o}$ es el valor resistivo de la impedancia equivalente de salida de la fuente piezoeléctrica presentada.

$$
P_{\text {RoutMAXX }}=\frac{V_{o c}^{2}}{4 \cdot R_{o}}
$$

Estos valores proceden de la caracterización de nuestra fuente mediante las gráficas de regulación de las figuras 10 y 12. Verificamos que la máxima transferencia de potencia se producirá cuando el valor de la resistencia que actúa como carga sea idéntico al de la resistencia equivalente de salida del circuito que hemos modelado, formado por todas las asociaciones de cables piezoeléctricos, las estructuras rectificadoras y la capacidad de acumulación de carga. También comprobamos que la impedancia equivalente de salida, debido a la amplia zona lineal que nos muestran las

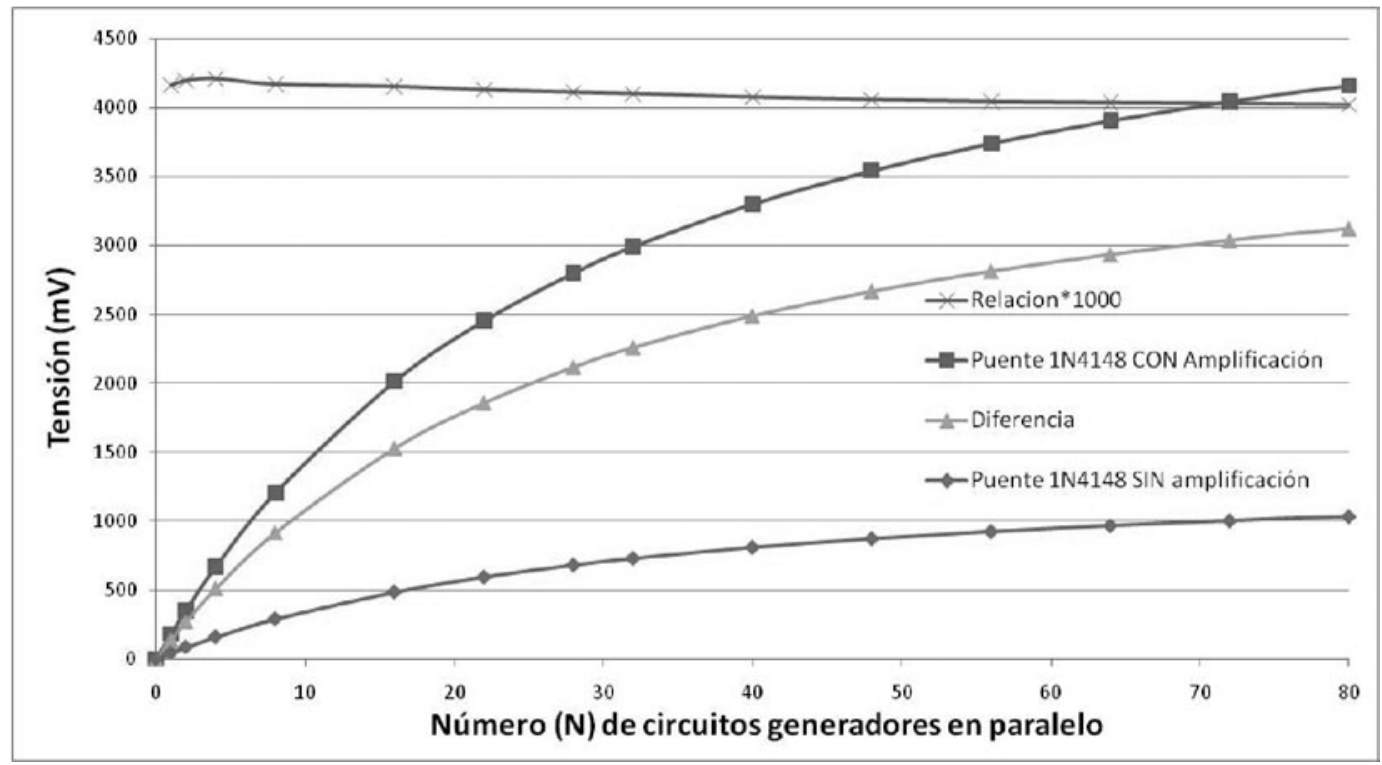

Figura 11: Tensión obtenida en el condensador de acumulación para los modelos correspondientes a vehículos ligeros (sin amplificación mecánica) y vehículos pesados (con amplificación) según el número $\mathrm{N}$ de circuitos rectificadores en paralelo. Relación (con factor *1000) y diferencia entre ambos. 


\begin{tabular}{|c|c|c|c|}
\hline ENSAYOS & $\mathrm{V}_{\mathrm{oc}}(\mathrm{mV})$ & $\mathrm{R}_{\mathrm{o}}(\Omega)$ & $\mathbf{P}_{\text {RoutMÁx }}(\mu \mathbf{W})$ \\
\hline Modelo SIN amplificación mecánica & $2.748,3$ & 715.431 & 2,63937 \\
\hline Modelo CON amplificación mecánica & 11.341 & 666.300 & 48,258 \\
\hline
\end{tabular}

gráficas de regulación, resulta un valor puramente resistivo, correspondiente con la ecuación 5.

$$
R_{o}=\frac{\Delta V_{o}}{\Delta I_{o}}
$$

Finalmente, en la tabla IV, se obtienen los valores al aplicar las ecuaciones 4 y 5 para las gráficas correspondientes a las curvas de regulación de las figuras 10 y 12. Dado que la resistencia equivalente de salida resulta ser muy próxima a $700 \mathrm{k} \Omega$ en ambos casos, validamos como los puntos de máxima potencia que anteriormente hemos extraído de estas gráficas son correctos ya que se corresponden con las simulaciones ejecutadas con ese valor de resistencia de carga. Los valores bajos de potencia máxima utilizable son clara consecuencia del problema práctico con el que nos enfrentamos, ya que trabajamos con una fuente de energía, en forma de tensión eléctrica continua, con una altísima resistencia equivalente de salida. Resultará interesante continuar con posteriores estudios para determinar maneras de elevar la tensión disponible $\mathrm{V}_{\text {oc' }}$ estudiando otros compuestos piezoeléctricos o variantes de excitación mecánica, y reflejar los valores de $R_{o}$.

\section{CONCLUSIONES.}

Se ha presentado en este trabajo el procedimiento de modelado eléctrico para un material de tipo piezoeléctrico, al que se ha sometido a excitación mecánica análoga a tráfico rodado, mediante la utilización del banco de ensayos, construido y diseñado para emular dicho entorno real. Aplicando el método expuesto a los resultados registrados de las correspondientes respuestas eléctricas, concluimos con la generación de modelos lineales que admiten su utilización aplicada a la generación de energía eléctrica para alimentación autónoma de sensores, transductores u otros dispositivos electrónicos diseñados para aplicaciones relacionadas con el tráfico vial o con las estructuras viales. Las conclusiones aplicables al estudio de los procesos de acumulación de energía eléctrica basados en este tipo de materiales son coherentes con el proceso de simulación eléctrico-electrónico empleado, permitiendo el cálculo del número de elementos necesarios y su configuración para obtener un determinado objetivo de alimentación. Este estudio se pretende ampliar próximamente mediante la utilización de la metodología descrita para la obtención de modelos eléctricos de otros dispositivos y materiales piezoeléctricos diferentes al utilizado hasta el momento, valorándose además la implementación en una vía de comunicación de un prototipo con el que ampliar conclusiones para el estudio iniciado sobre generación de energía eléctrica de forma autónoma.

\section{AGRADECIMIENTOS}

Este trabajo se ha financiado con el proyecto MAT201021088-C03-03
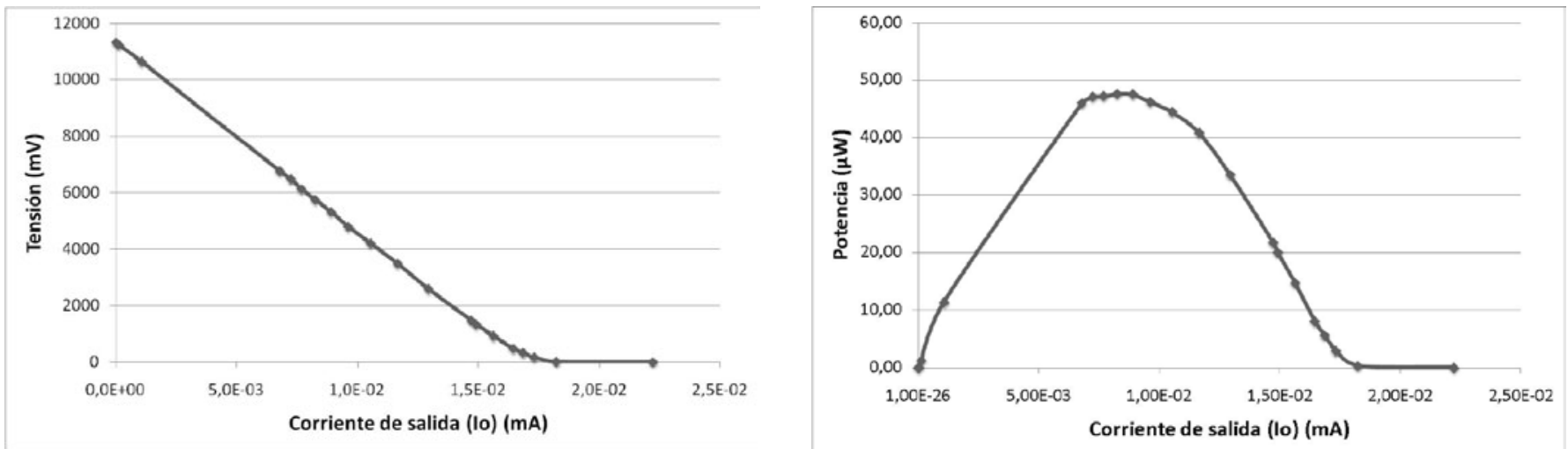

Figura 12: Resultados para tráfico pesado de la asociación de 2 circuitos serie de $\mathrm{N}=80$ generadores paralelo cada uno: (a) Tensión disponible según la corriente consumida, (b) Potencia disponible en función de la corriente consumida. 


\section{BIBLIOGRAFÍA}

1. Beeby, S. P.; Torah, R. N.; Tudor, M. J.; Glynne-Jones, P.; ODonnell, T; Saha, C. R. \& Roy, S. A micro electromagnetic generator for vibration energy harvesting, J. Micromech. Microeng., Vol. 17, 1257-1265. (2007).

2. Cook-Chennault, K. A., Thambi, N., \& Sastry, A. M. Powering MEMS portable devices $-A$ review of nonregenerative and regenerative power supply systems with special emphasis on piezoelectric energy harvesting systems. Smart Materials \& Structures, 17 (4) (2008)

3. K. A. Cook-Chennault; N. Thambi; M.A. Bitetto; E.B. Hameyie Piezoelectric Energy Harvesting: A Green and Clean Alternative for Sustained Power Production. Bulletin of Science, Technology \& Society, vol. 28, 6: pp. 496509. (2008)

4. Miles, R. W., Hynes, K. M., \& Forbes, I. Photovoltaic solar cells: An overview of state-of-the-art cell development and environmental issues. Progress in Crystal Growth and Characterization of Materials, 51 (1-3), 1-42. (2005).

5. Mitcheson, P. D., Reilly, E. K., Toh, T., Wright, P. K., \& Yeatman, E. M. Performance limits of the three MEMS inertial energy generator transduction types. Journal of Micromechanics and Microengineering, 17 (9), S211-S216 (2007)

6. O'Neill, M. J., Piszczor, M. F., Eskenazi, M. I., McDanal, A. J., George, P. J., Botke, M. M., et al. The stretched lens array (SLA): A low-risk, costeffective concentrator array offering wing-level performance of $180 \mathrm{~W} /$ $\mathrm{kg}$ and $300 \mathrm{w} / \mathrm{m}(2)$ at 300 VDC. IEEE Aerospace and Electronic Systems Magazine, 18 (1), 3-9 (2003)

7. Shearwood, C., \& Yates, R. B. Development of an electromagnetic microgenerator. Electronics Letters, 33 (22), 18831884 (1997).

8. Xue, J. G., Uchida, S., Rand, B. P., \& Forrest, S. R. $4.2 \%$ efficient organic photovoltaic cells with low series resistances. Applied Physics Letters, 84 (16), 3013-3015. (2004).

9. Sodano, H. A., Lloyd, J., \& Inman, D. J. An experimental comparison between several active composite actuators for power generation. Smart Materials \& Structures, 15 (5), 1211-1216. (2006)

10. Priya, S., Chen, C. T., Fye, D., \& Zahnd, J.. Piezoelectric windmill: A novel solution to remote sensing. Japanese Journal of Applied Physics: Part 2Letters \& Express Letters, 44 (1-7), L104-L107. (2005)

11. Shenck, N. S., \& Paradiso, J. A. Energy scavenging with shoe-mounted piezoelectrics. IEEE Micro, 21 (3), 30-42. (2001).

12. Schmidt, V. H. Piezoelectric energy conversion in windmills. IEEE Ultrasonic Symposium, pp. 897-904. (1992).

13. Ramsay, M. J., \& Clark, W. W. Piezoelectric energy harvesting for bio MEMS applications. Smart Structures and Materials, Industrial Proceedings of SPIE. (2001)

14. Platt, S. R., Farritor, S., Garvin, K., \& Haider, H. The use of piezoelectric ceramics for electric power generation within orthopedic implants. IEEEASME Transactions on Mechatronics, 10 (4), 455-461. (2005).

15. Jeon Y. B., Sood R., Jeong J. H., Kim S. G. MEMS power generator with transverse mode thin film PZT, Sensors and Actuators A: Physical, 122(1), 16-22. (2005).
16. White, N. M., Glynne-Jones, P., \& Beeby, S. P. A novel thick-film piezoelectric micro-generator. Smart Materials \& Structures, 10 (4), 850852. (2001)

17. Roundy, S., \& Wright, P. K. A piezoelectric vibration based generator for wireless electronics. Smart Materials \& Structures, 13 (5), 1131-1142. (2004)

18. Whalen, S., Thompson, M., Bahr, D., Richards, C., \& Richards, R. Design, fabrication and testing of the P 3 micro heat engine. Sensors and Actuators A: Physical, 104 (3), 290-298. (2003)

19. Kim H. W., Batra A., Priya S., Uchino K., Markley D., Newnham R. E., Hofmann H. F. Energy harvesting using a piezoelectric "cymbal" transducer in dynamic environment. Jap. Jour. Appl. Phys. 43, 6178-6183. (2004).

20. Engel, T. G., Keawboonchuay, C., \& Nunnally, W. C. Energy conversion and high power pulse production using miniature piezoelectric compressors. IEEE Transactions on Plasma Science, 28 (5), 1338-1341. (2000)

21. Ren, K. L., Liu, Y. M., Geng, X., Hofmann, H. F., \& Zhang, Q. M.. Single crystal PMN-PT/epoxy 1-3 composite for energy harvesting application. IEEE Transactions on Ultrasonics, Ferroelectrics, and Frequency Control, 53 (3), 631-638. (2006)

22. Sheng Wang, Kwok Ho Lam, Cheng Liang Sun, Kin Wing Kwok, Helen Lai Wa Chan, Ming Sen Guo, and Xing-Zhong Zhao Energy harvesting with piezoelectric drum transducer Appl. Phys. Lett. 90, 113506 (2007)

23. Zhong Lin Wang, Jinhui Song; Piezoelectric Nanogenerators Based on Zinc Oxide Nanowire Arrays; Sience Vol. 312 no. 5771 pp. 242-246 (2006)

24. Chung-Bang Yun and Jiyoung Min Smart Sensing, Monitoring, and Damage Detection for Civil Infrastructures KSCE Journal of Civil Engineering 15(1):1-14 (2011)

25. N. Elvin, A. Elvin, D.H. Choi. A self-powered damage detection sensor. J. Strain Anal. 38 (2) 115-124. (2003)

26. M. Vázquez Rodríguez, F.J. Jiménez Martínez, J. de Frutos. Banco de ensayos para materiales piezoeléctricos en aplicaciones viales. Bol. Soc. Esp. Ceram. Vidr. Vol 50. 2, 65-72. (2011)

27. Zhu M, Worthington E and Njuguna J. Analyses of power output of piezoelectric energy-harvesting devices directly connected to a load resistor using a coupled piezoelectric-circuit finite element method. IEEE Trans. Ultrason. Ferroelectr. Freq. Control 56 1309-17. 2009.

28. J. Sun, D. M. Mitchell, M. F. Greuel, and R. M. Bass, Averaged modeling of PWM converters in discontinuous conduction mode. IEEE Trans. Power Electron., vol. 16, pp. 482-492, July 2001.

29. N. Mohan, T. Undeland, and W. Robbins, Power Electronics: Converters, Applications and Design. New York: Wiley, 1995

30. G. K. Ottman, A. C. Bhatt, H. Hofmann, and G. A. Lesieutre, Adaptive piezoelectric energy harvesting circuit for wireless remote power supply, IEEE Trans. Power Electron., vol. 17, pp. 669-676, Sept. 2002.

31. G.K. Ottman, H.F. Hofmann, G.A. Lesieutre. Optimized piezoelectric energy harvesting circuit using step-down converter in discontinuous conduction mode. IEEE Trans. Power Elec. 18 (2) 696-703. (2003) 